\title{
NDT of Reinforcement Corrosion Using Ultrasonic Spectroscopy
}

\author{
M. Kořenská*, M. Matysík \\ Department of Physics, Faculty of Civil Engineering, Brno University of Technology, Brno, Czech Republic \\ *Corresponding author: korenska.m@fce.vutbr.cz
}

K. Pospíšil

CDV - Transport Research Centre, Brno, Czech Republic

\begin{abstract}
Corrosion of built-in steel reinforcement ranks among the most serious mechanisms of bridge structure degradation. There are many reasons for the corrosion to occur: failures occurring during the bridge construction, consequences of traffic load, or, simple, ageing of the structures. Visual inspection of a bridge provides general information on the bridge condition. However, it cannot provide any information on the internal structure and integrity of the reinforced concrete or pre-loaded elements of the bridge in question.

This is why non-destructive diagnostic methods are acquiring growing importance, helping researchers to properly evaluate the condition of a bridge and decide upon the most convenient methods for maintenance, repair or refurbishment of the bridge in question or its parts and schedule them accordingly.

In this domain, methods employing the non-linear acoustic spectroscopy (NEWS Nonlinear Elastic Wave Spectroscopy) achieved rush advancement recently. They are based on the fact that a non-linearity, which is due to the presence of a defect, makes an extraordinary indicator of the structure damage. These new, non-destructive methods appear to be promising for application to a wide range of materials featuring relatively heavy non-homogeneities, and for a large span of sites, from micro-chip to bridge structures.

The present paper deals with an experimental study of the application of non-linear ultrasonic spectroscopy methods to the detection of steel reinforcement corrosion and its consequences for reinforced concrete specimens subjected to corrosion induced degradation cycles.
\end{abstract}

KEY WORDS: Reinforced concrete, reinforcement corrosion, nonlinear ultrasonic spectroscopy, nonlinear effects

\section{INTRODUCTION}

Bridge structures make up an important element of traffic infrastructure. In most cases they consist of reinforced or preloaded concrete structures originating from the second half of last century. Concrete proved to be a durable construction material in the past. However, concrete structures often experience degradation after years of service. One of the frequent breakdown causes consists in the steel reinforcement corrosion. There are cases where a bridge structure collapsed in consequence of the reinforcement corrosion. Redevelopment technologies have been experiencing a marked growth in foreign countries during last decades. In this country, thanks to the efforts of a number of renowned experts and professional societies a rapid development has come to fruition in this field, too. In this way, our building industry has almost reached the foreign standard. However, the absence of an acceptable, relatively fast and cheap monitoring method, which would 
be capable of detecting bridge faults at an early stage, thus making a simple and cost-effective maintenance possible, is still persisting. This is why great attention is paid to the design and testing of new non-destructive methods meeting the above mentioned requirements.

\section{NONLINEAR ULTRASONIC SPECTROSCOPY}

New, promising, non-destructive testing methods are based on the non-linear behaviour of current defects and inhomogeneities regarding the elastic wave propagation processes. There are two groups of methods available for application: resonance and non-resonance. Bodies exhibiting strong resonance effects make it possible to study, above all, the non-linear effect of the resonance frequency shift versus exciting signal intensity. These methods are usually referred to as SIMONRAS (Single Mode Nonlinear Resonance Ultrasound or Acoustic Spectroscopy) (K. Van Den Abeele et. al., 2000, R. G. Litwiller, 2002). The resonance methods are rather labour-intensive and require many readings to be taken (frequency response curves for various signal magnitude levels). Therefore, they are not suited for fast in-process measurements.

Non-resonance methods are used to study suppressed resonance specimens. These methods analyse the effect of non-linearities on acoustic signals propagating through them. These methods can be split into two groups. In the first group, a single ultrasound harmonic signal is employed. The non-linearity gives rise to additional signals featuring different frequencies according to a Fourier expansion. In general, the amplitudes of these additional components decrease with the natural number $n$ :

$$
\mathrm{f}_{\mathrm{n}}=n \mathrm{f}_{1} \quad \mid n=0,1,2 . . \infty,
$$

Nevertheless, among the emerged signals, the third harmonic appears to be most pronounced, see Fig. 1. This is why the third harmonic amplitude is pursued by most researchers, especially in electronics (Hajek et. al., 2003).
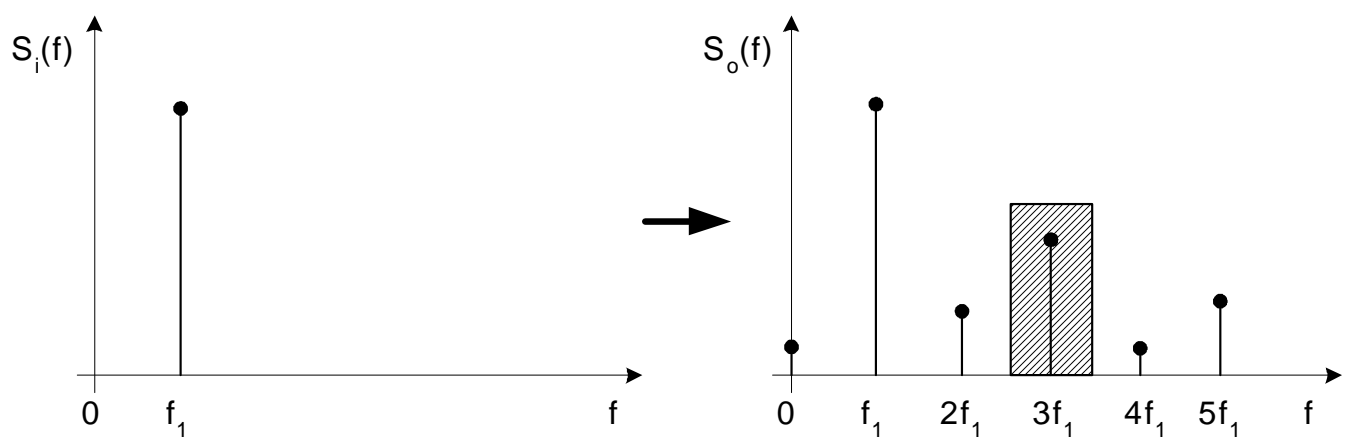

Figure 1: Growth higher harmonic components in frequency spectra at transit pure harmonic signal through nonlinear environment with illustration of selection dominant third harmonic component by the frequency band-pass filter.

In the second case, several (usually two) ultrasound signals are applied to the specimen. The number of additional harmonic components generated is substantially higher. 
In addition to both exciting signals' harmonics, one also gets sum and difference frequency components.

$$
\mathrm{f}_{\mathrm{v}}=\left| \pm \mathrm{m} \mathrm{f}_{1} \pm \mathrm{nf}_{2}\right| \quad \mid \mathrm{m}, \mathrm{n}=0,1,2 . . \infty
$$

Owing to the general harmonic amplitude versus frequency curve downward slope, the first sum and difference components are most pronounced. The application domain of the ultrasound modulation spectroscopy (usually referred to as NWMS - Nonlinear Wave Modulation Spectroscopy) splits into two subgroups, which differ from each other by the exciting frequency ratio. Attention is paid to the second subgroup applied in experimental part. In this case, the frequency mixing principle is used. The signal frequencies are close to each other. The first difference component therefore falls into the low-frequency range, as is shown in Fig. 2.

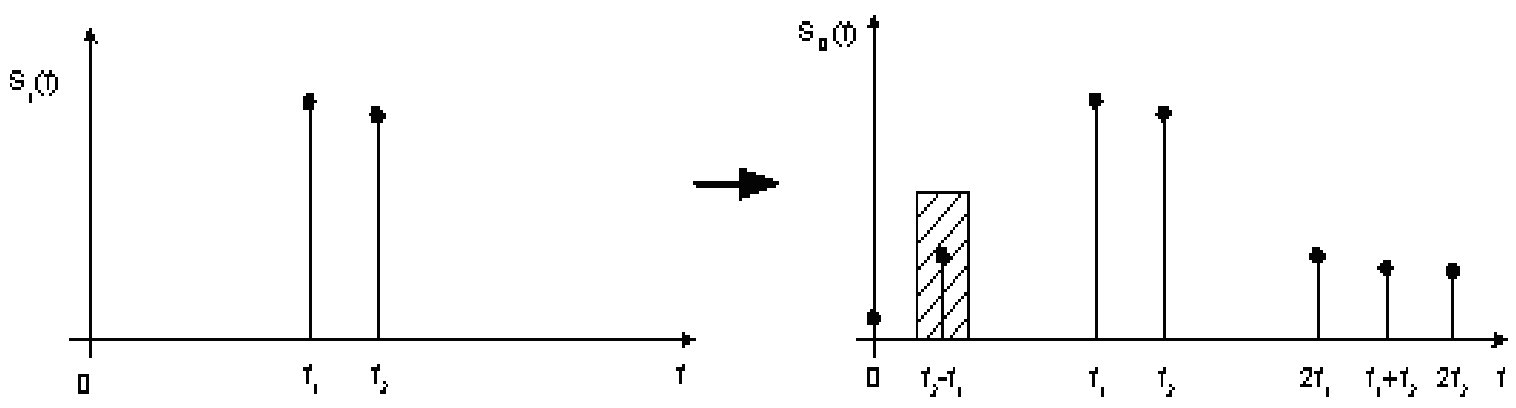

Figure 2: Creation of new harmonic components in frequency spectra at transit two harmonic signal through nonlinear environment with demonstration of selection dominant components by frequency filter - event of mixing (with small rate of $f_{2} / f_{1}$ )

Recently, various papers on both the theoretical and experimental examination of diverse methods and their applicability in some fields have been published. Most published papers, as well as our experience, show these methods to be highly promising for the defectoscopy and the material testing purposes in the near future.

One of the fields in which a wide application range of non-linear acoustic spectroscopy methods may be expected is civil engineering. Poor material homogeneity, and, in some cases, the shape complexity of some units used in the building industry, are heavily restricting the applicability of "classical" ultrasonic methods (Macecek, 2003). Precisely these non-linear acoustic defectoscopy methods are less susceptible to the mentioned restrictions and one may expect them to contribute a great deal to further improvement of the defectoscopy and material testing in civil engineering.

\section{TESTED OBJECT AND EXPERIMENTAL ARRANGEMENT}

Reinforced concrete joists of atypical dimensions of $50 \mathrm{~mm} \times 50 \mathrm{~mm} \times 360 \mathrm{~mm}$, containing a smooth steel bar of a diameter of $8 \mathrm{~mm}$ located in the joist's longitudinal centre line, were studied in this experiment. Two mutual orientations of the ultrasonic transmitter - sensor connecting line with respect to the specimen longitudinal centre line were selected for the measurements, namely, the transversal orientation a), and the longitudinal orientation 
b) as is shown in Fig. 3. A set of six specimens in a degradation process stage plus a set of three reference specimens were tested.

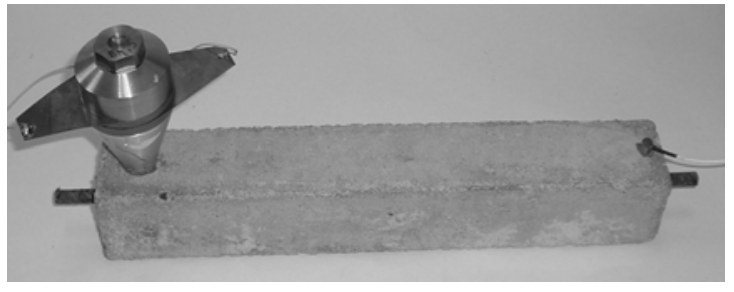

a) Transversal orientation

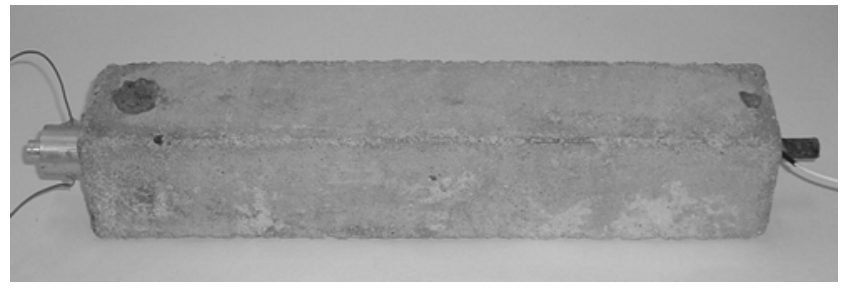

b) Longitudinal orientation

Figure 3: Location of the exciter and the sensor on the specimen under testing

In the first measurement stage, a single harmonic ultrasonic signal method was applied. The experimental set-up and testing of its component units have been described in detail previously (Manychova, 2007) and will only be briefly described here, see Fig. 4.

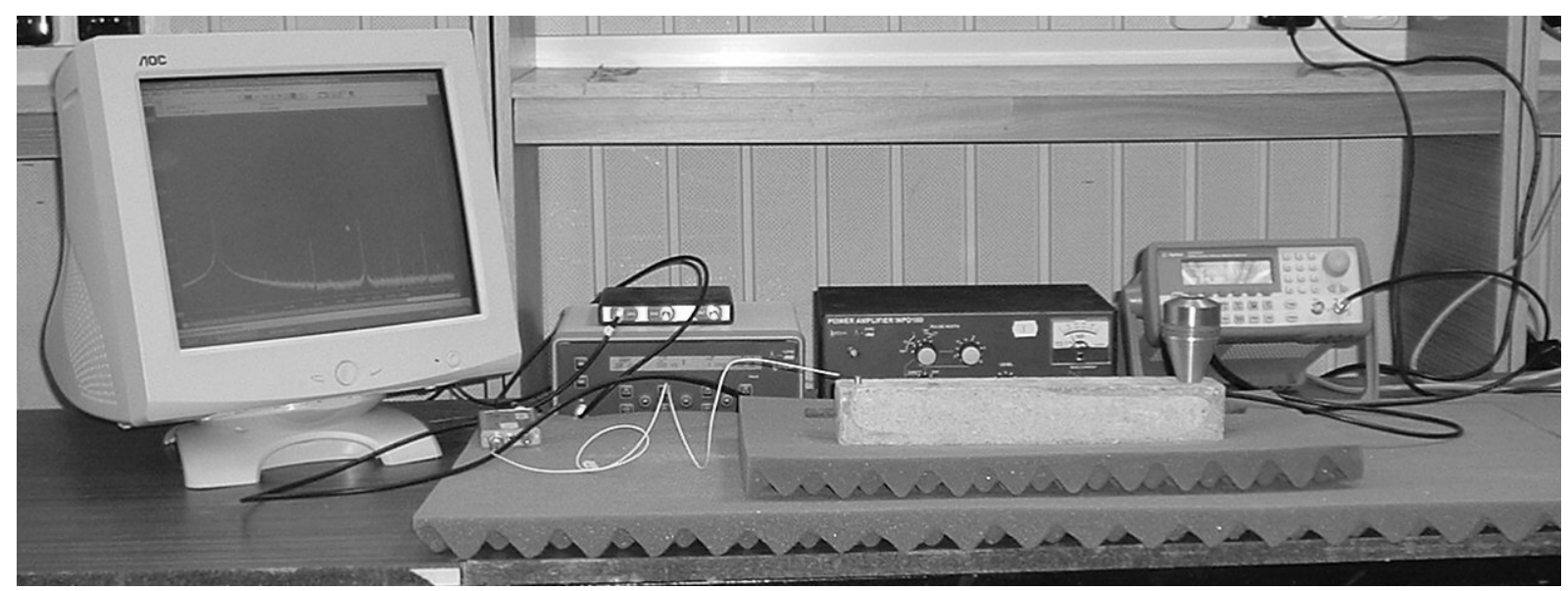

Figure 4: Experimental arrangement, photo of the nonlinear ultrasonic equipment with a test specimen

The measuring apparatus consists of two principal parts, namely, a transmitting section and a receiving and measuring section. The transmitting section consists of four functional blocks: a controlled-output-level harmonic signal generator, a low-distortion $100 \mathrm{~W}$ power amplifier, and an output low-pass filter to suppress higher harmonic components and ensure a high purity of the exciting harmonic signal. The main chain of the receiving section includes an input amplifier with filters designed to minimize the receiving chain distortion and a bandpass filter amplifier. Having been amplified, the sensor output signal was fed into a THPS3-25 HandyScope3 measuring instrument to be sampled and analyzed. For the purpose of improving the reliability and accuracy of the nonlinear experiments, and minimizing the error effects, attention was focused on transmission between exciter and sensors. Elements meeting the given requirements were chosen (Korenska et. al., 2006). A program package to control the measuring process, the data processing and evaluation makes an indispensable tool. The measurement results were represented in the form of frequency spectra.

In the second stage of the experiment, a double-signal non-linear ultrasonic spectroscopy was applied, see Fig. 2. In the case of our experiment, the frequency difference fell into a frequency range below $5 \mathrm{kHz}$. From the relatively high difference between the exciting signal frequencies and the difference component frequency there results a distinctive 
advantage of directly detecting this difference component, provided that an analog highdynamic-range (up to over $120 \mathrm{~dB}$ ) pre-filtering network is used.

\section{EXPERIMENT RESULTS}

Generation of higher harmonic frequencies for an exciting frequency of $f=29 \mathrm{kHz}$ was studied in the first stage of the experiment. The measurement results can be expressed in the form of frequency spectra, as is shown in the following Figures. Measurement results obtained from reference specimens are represented by frequency spectra of specimen No. 05TP8C0, Fig. 5.

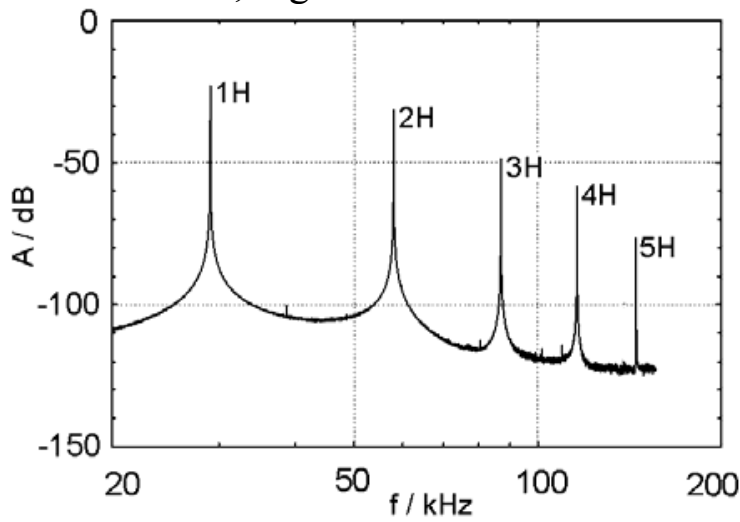

a) Transversal orientation

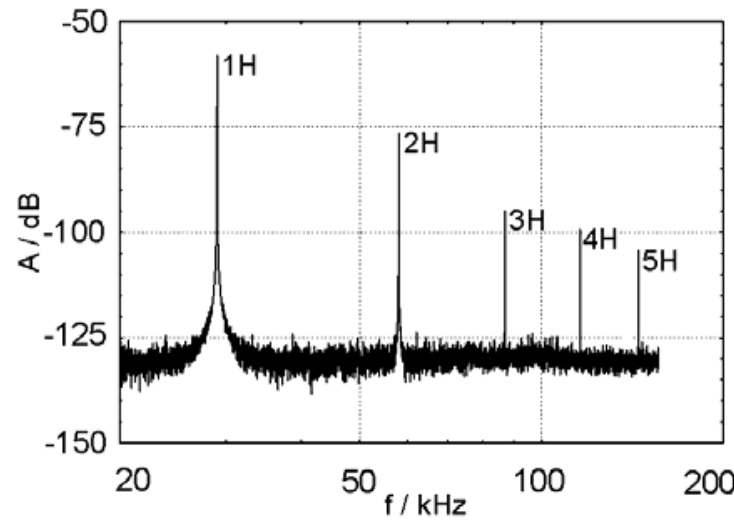

b) Longitudinal orientation

Figure 5: 05TP8C0 reference specimen

The spectrum of Fig. 5a) shows the measurement results obtained from this specimen measured under the conditions of transversal orientation. Higher harmonic amplitudes are decreasing with their increasing serial number. The surface area available for fitting the transmitter onto the specimen was restricted by the steel reinforcement in the case of longitudinal excitation. Therefore, a smaller-sized high frequency exciter was used. The longitudinal orientation results are shown in Fig. 5b). This frequency spectrum shows again a drop of higher-frequency amplitudes with an increasing serial number. Lower amplitudes, which were measured in the longitudinal orientation conditions, are due to the lower output of the HF exciter at the exciting frequency $29 \mathrm{kHz}$.

Measurement results obtained from the specimens which were degraded in 82 corrosion cycles are represented by frequency spectrum of specimen No. 10TP8C82, see Fig. 6.

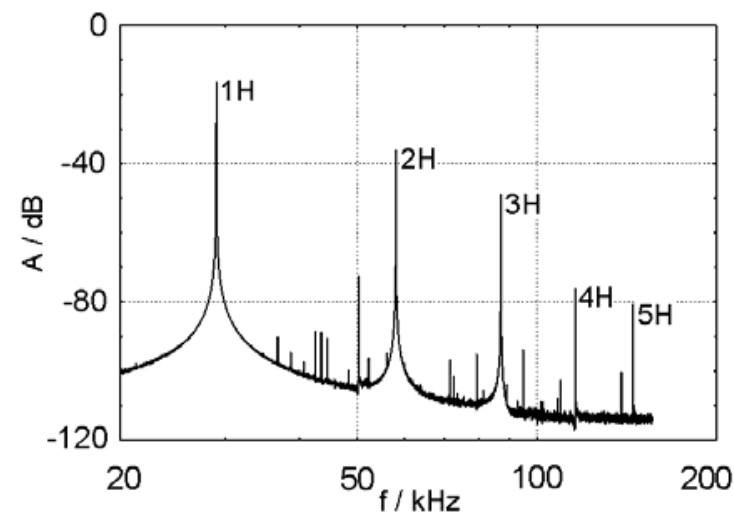

a) Transversal orientation

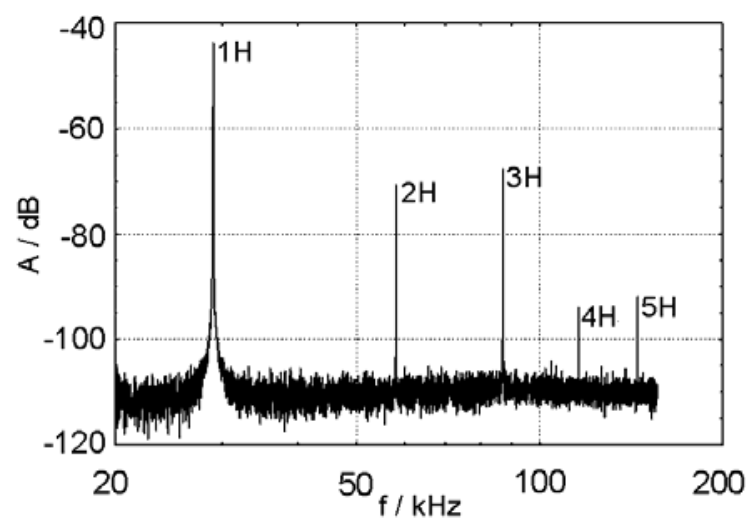

b) Longitudinal orientation

Figure 6: $10 \mathrm{TP8C} 82$ specimen - after 82 corrosion cycles 
The frequency spectrum of Fig. 6a) corresponding to the transversal orientation contains not only the harmonic frequencies, but also some other frequency components, whose amplitudes are comparable with those of the fourth $(4 \mathrm{H})$ and fifth $(5 \mathrm{H})$ harmonic frequency. The same effect was observed in concrete and light-concrete joists after they had been stressed in a pressing machine until a visible crack appeared (Matysik et. al., 2007, Korenska et. al. 2008). The longitudinal-orientation transfer function curve differs from that of the intact specimen, see Fig. 6b). In the frequency spectrum the amplitudes of the odd-numbered harmonic frequency components $3 \mathrm{H} / 5 \mathrm{H}$ exceed those of the evennumbered ones $2 \mathrm{H} / 4 \mathrm{H}$.

The next Fig. 7 and 8 represented the results of our measurement when two ultrasonic signals $f_{1}=32 \mathrm{kHz}, f_{2}=29 \mathrm{kHz}$ have been applied to specimens. A difference component of a frequency $f_{v}=3 \mathrm{kHz}$ was looked for. The chart of Fig. 7 corresponds to reference specimens.

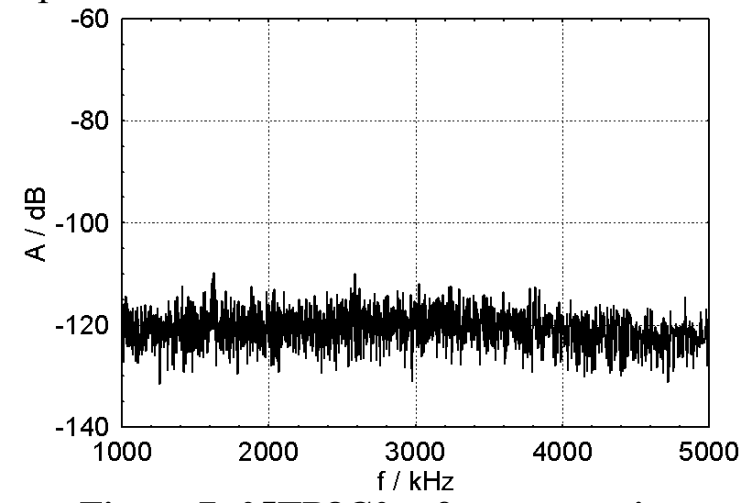

Figure 7: 05TP8C0 reference specimen

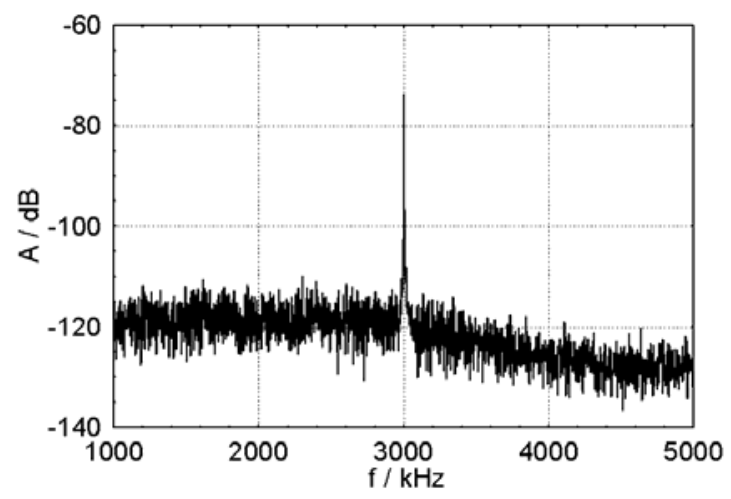

Figure 8: 10TP8C82 specimen - 82 cycles

It is clear from the diagram that no inter-modulation of the two ultrasonic signals takes place, which is evidence of the structure integrity of the specimen under test being intact. Measurement results of a specimen after 82 degradation cycles are represented in Fig. 8 . The predominating magnitude of the amplitude occurring at this difference component is due to the presence of a defect in consequence of reinforcement corrosion.

Further to the above mentioned measurements, X-ray photographs of the 05TP8C0 reference specimen and the 10TP8C 82 specimen (after 82 corrosion cycle application) were taken, see Fig. 9. The upper photograph, denoted 08 and corresponding to the reference specimen, clearly shows that the reinforcement-to-concrete bond has remained intact. The lower photograph, denoted 8, illustrates the corroded specimen. In consequence of the corrosion, the reinforcement-to-concrete bond has been disturbed at several points along the reinforcement. One of the damaged areas is shown in the photograph.

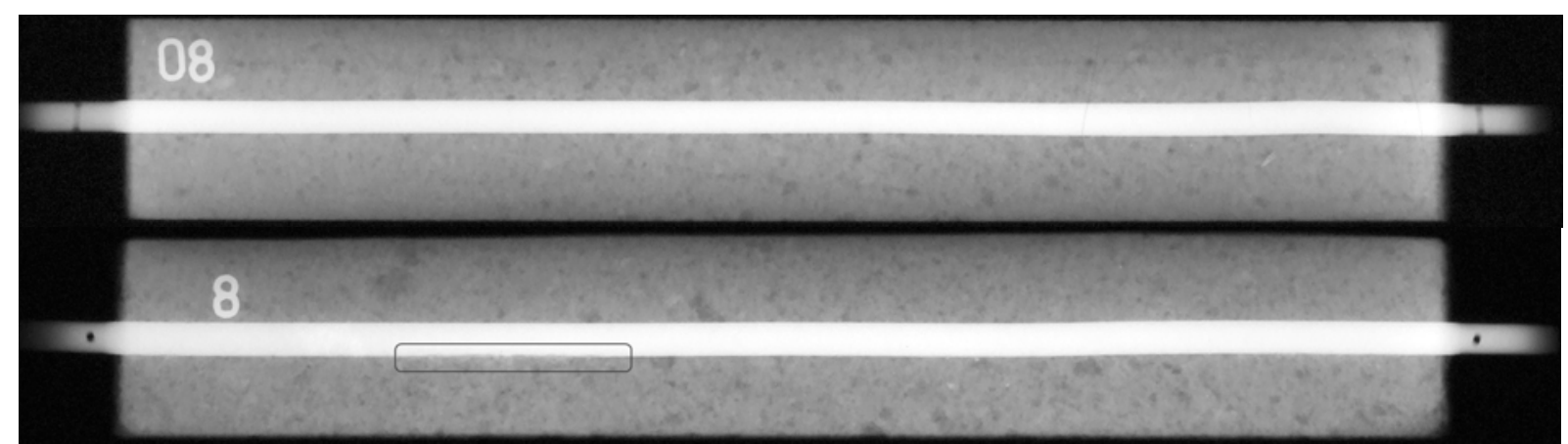

Figure 9: X-ray photographs of 05TP8C0 reference specimen (08) and 10TP8C82 specimen after 82 degradation cycles application (8). 


\section{CONCLUSION}

In the first stage of the experiment, with a single-frequency harmonic exciting signal, it has been proved that the frequency spectra of reference (non-corroded) specimens do not exhibit non-linearities in the signal transmission. On the other hand, transfer characteristics of the specimens measured after the application of 82 corrosion cycles do show non-linear effects, which correlate with the steel armature corrosion consequences.

These non-linear effects are apparent for both transversal and longitudinal excitation arrangements from the relatively high harmonic amplitude behaviour. The respective amplitudes do not show the gradual decrease with the increasing harmonic order number, as is the case of the reference specimens. Moreover, the distorted structure was shown to generate non-harmonic frequency components in the case of transversal excitation.

When a double-frequency exciting signal method was applied, the consequences of the corrosion gave rise to a large-amplitude component whose frequency equalled to the exciting frequency difference.

The following conclusions have been drawn from the application of the above mentioned methods:

The single harmonic signal exciting method provides very sensitive detection and accurate defect localization. It, however, requires - particularly in the case of bulky specimens a high exciting power, which is rather difficult to generate with the required spectrum purity.

The double harmonic signal modulation method, using an exciting signal whose frequencies near each other, does not require a pure signal spectrum, although it requires a certain degree of the source linearity - to minimize mutual non-linear interaction. A certain drawback of this method consists in the rather low frequency of the exciting signal, which in its turn lowers the localisation potential of this method.

In conclusion, it is to be noted that a mechanical impulse exciting signal carries a much higher power than any pure harmonic signal electric excitation. The measurement sensitivity of such a measuring method is much higher. These methods are especially suitable for pronounced resonance response exhibiting specimens, where the broad-band impulse excitation results virtually in a narrow-band or even harmonic response. A typical example of pulse excitation used in everyday practice is a spectral analysis carried out by a human ear during a hammer-tap test of a railway wagon wheel, or the change in the sound spectrum of a broken bell. Beside the generation of new harmonic components, the defect induced nonlinearity also results in a change of the specimen transfer characteristics and both phenomena can be analysed at a time.

\section{ACKNOWLEDGMENTS}

The research described in this paper was established within the scope of the research project of the Grant Agency of the Czech Republic under contract No. 103/06/1711.

The authors would like to thank Mr. Assoc. prof. Hobst for the X-ray photographs of specimens and Mr. Skala for preparing the specimens for observation. 


\section{REFERENCES}

Abeele, K. V. D., Carmeliet, J., Cate, J. A. T., Johnson, P. A., 2000. Nonlinear Elastic Wave Spectroscopy (NEWS) Techniques to Discern Material Damage. Part II: Single Mode Nonlinear Resonance Acoustic Spectroscopy. Res. Nondestr. Eval. 12/1, pp. 31 - 42, 2000, http://perswww.kuleuve.ac.be/ u0004811/simonras.pdf.

Litwiller, R. G., 2002. Resonant Ultrasound Spectroscopy and the Elastic Properties of Several Selected Materials. http://www.kristall.ethz.ch/rigi2002/abstracts/abstractleisure.pdf.

Hajek, K., Sikula J., 2003. Testing of Low-Current Contacts Quality and Reliability by Using Third Harmonic Distortion. Proc. of 45th- IEEE Holm Conference, Washington, September 2003, pp. 211 - 213. ISBN 0-7803-7862-8.

Marecek, M., 2003. Ultrasonic Concrete Testing, Defektoskopie 2003, 33rd International Conference, November 19-21, 2003, Ostrava, Czech Republic, pp. 117 - 132, ISBN 80-214-2475-3.

Manychova, M., 2007. Measuring Setup for the Nonlinear Ultrasonic Spectroscopy Method. In Defektoskopie 2007, edited by M. Korenska et al., 37th International Conference Proceedings, Prague, 2007, pp. 147 - 151.

Korenska, M., Pazdera, L., Matysik, M., 2006. Testing of Pickups for Single-exciting-signal Non-linear Acoustic Spectroscopy. In Physical and Material Engineering 2006, International Workshop Proceedings, Slovak University of Technology in Bratislava, September 2006, pp. 70 - 75.

Matysik, M., Korenska, M., Plskova, I., Kucharczykova, B., Keprt, J., 2007. Application of the Non-linear Ultrasonic Spectroscopy Method to Lightweight Concrete Specimen Testing. In Proceedings of 5thWorkshop NDT 2007, Non-destructive Testing in Engineering Practice, November 29, 2007, Brno, ISBN 978-80-7204-549-5, pp. 82 - 87.

Korenska, M., Matysik, M., Plskova, I., Manychova, M., 2008. Monitoring of Concrete Components Structure Condition Using a Nonlinear Ultrasonic Spectroscopy. Proceedings of the 17th World Conference on Non-destructive Testing, Shanghai, p. 6, CD, The e-Journal of Non-destructive Testing, ndt.net, Germany, pp. 1 - 6, ISSN 1435 4934. 